\title{
Different Modes of Pitch Perception and Learning-Induced Neuronal Plasticity of the Human Auditory Cortex
}

\author{
Michael Schulte, ${ }^{1,2}$ Arne Knief, ${ }^{1,2}$ Annemarie Seither-Preisler ${ }^{1}$ and Christo Pantev ${ }^{2}$ \\ ${ }^{1}$ Biomagnetism Center, Institute of Experimental Audiology, University of Münster, 48129 \\ Münster, Germany; ${ }^{2}$ Rotman Research Institute, Baycrest Centre for Geriatric Care, \\ University of Toronto, Ontario, Canada
}

\begin{abstract}
SUMMARY
We designed a melody perception experiment involving eight harmonic complex tones of missing fundamental frequencies (hidden auditory object) to study the short-term neuronal plasticity of the auditory cortex. In this experiment, the fundamental frequencies of the complex tones followed the beginning of the virtual melody of the tune "Frère Jacques". The harmonics of the complex tones were chosen so that the spectral melody had an inverse contour when compared with the virtual one. Evoked magnetic fields were recorded contralaterally to the ear of stimulation from both hemispheres. After a base line measurement, the subjects were exposed repeatedly to the experimental stimuli for 1 hour a day. All subjects reported a sudden change in the perceived melody, indicating possible reorganization of the cortical processes involved in the virtual pitch formation. After this switch in perception, a second measurement was performed. Cortical sources of the evoked gamma-band activity were significantly stronger and located more medially after a switch in perception. Independent Component Analysis
\end{abstract}

Reprint requests to: Christo Pantev, PhD, D.Sc., Canada Research Chair "Human Cortical Plasticity", University of Toronto, Rotman Research Institute, Baycrest Centre for Geriatric Care, 3560 Bathurst Street, Toronto, Ontario, Canada M6A 2E1 revealed enhanced synchronization in the gammaband frequency range. Comparing the gammaband activation of both hemispheres, no laterality effects were observed. The results indicate that the primary auditory cortices are involved in the process of virtual pitch perception and that their function is modifiable by laboratory manipulation.

\section{KEYWORDS}

magnetoencephalography (MEG), evoked gamma band, virtual pitch, Independent Component Analysis (ICA), hemispheric lateralization

\section{INTRODUCTION}

The sum of sinusoidal sound waves with frequencies that are integer multiples of a certain fundamental frequency $\left(\mathrm{f}_{0}\right)$ is known as a harmonic complex tone. Examples of such complex tones are vowels and tones produced by the human voice and most musical instruments. Basically, complex tones can be perceived according to their spectral characteristics or according to the pitch of their $\mathrm{f}_{0}$ (Smoorenburg, 1970; Terhardt, 1974; Hartmann, 1988). In the first case, single harmonics of a complex tone can be heard separately as distinct spectral pitches or they can fuse into a common 'spectral pitch' in the frequency region of the 
involved harmonics. In most cases, however, the latter type of perception is dominant and has been found to be independent of the physical presence of $\mathrm{f}_{0}$. Thus, it has been termed "pitch of the missing fundamental frequency" or "virtual pitch". The information about the harmonics has to be bound together into a coherent percept to extract the $\mathrm{f}_{0}$. This auditory binding is often compared with Gestalt recognition in the visual modality (Terhardt, 1974).

Gamna-band oscillations, as determined by electroencephalography (EEG) or magnetoencephalography (MEG), have often been found to be associated with integrative cognitive functions during object recognition. During the perception of a 'Gestalt', the different characteristics of an object are combined together into a coherent percept. Oscillatory neural discharges in the gamma frequency range have been proposed to be a representation of this binding process (Singer \& Gray, 1995). The frequency of the gamma-band responses is typically between 30 and $70 \mathrm{~Hz}$ for the auditory and visual modalities. Gamma-band responses (GBR) can be divided into two different types: evoked and induced (Pantev, 1995). Evoked activity is time- and phase-locked to the stimulus, whereas induced gamma-band activity is not. An enhancement of the spectral content of the induced gamma-band activity has been reported at latencies around $200 \mathrm{~ms}$ after stimulus onset in the perception of coherent versus non-coherent visual objects (Tallon-Baudry et al., 1996a; 1997), word detection tasks (Pulvermüller et al., 1996), and during shortterm memory experiments (Bertrand, 1998; TallonBaudry et al., 1998).

In contrast to induced gamma-band activity, the earlier transient evoked gamma-band activity with maximal amplitude around $60 \mathrm{~ms}$ after stimulus onset has been attributed to elementary perceptual processes (Pantev et al., 1991). The latencies of the auditory evoked gamma-band activity and their equivalent sources, as determined from EEG and MEG, suggest an origin in primary auditory cortical areas (Pantev \& Elbert, 1994; Pantev et al., 1991). Additional to this relatively early brain response, the $\mathrm{N} 1 \mathrm{~m}$ component of the slow auditory evoked field appears with latency of around $100 \mathrm{~ms}$ after the stimulus onset. Because $\mathrm{N} 1 \mathrm{~m}$ is most likely generated in secondary auditory structures, we have the chance to examine two different successive stages within the auditory cortex. Furthermore, this study was designed to answer the question if it is possible to learn to perceive the virtual pitch instead of the spectral one. By performing two measurement sessions, one before and one after the switch in perception, we were able to investigate the effect of use-dependent cortical plasticity as well as the process underlying the auditory binding in different stages of auditory processing. An additional point of interest was the lateralization of virtual-pitch processing and the underlying cortical sources.

\section{EXPERIMENTAL}

\section{Subjects}

Ten subjects (six males and four females), between 23 and 32 years of age, participated in this study. According to the Edinburgh handedness questionnaire (Oldfield, 1971), all subjects were right handed and had no history of otological or neurological disorders. After the nature of the study was explained, informed consent was obtained. Experimental procedures were in accordance with the Ethics Commission of the University of Münster and the Declaration of Helsinki.

\section{Stimulation}

The auditory stimulus was an eight-tone sequence, built up of three different complex tones. 


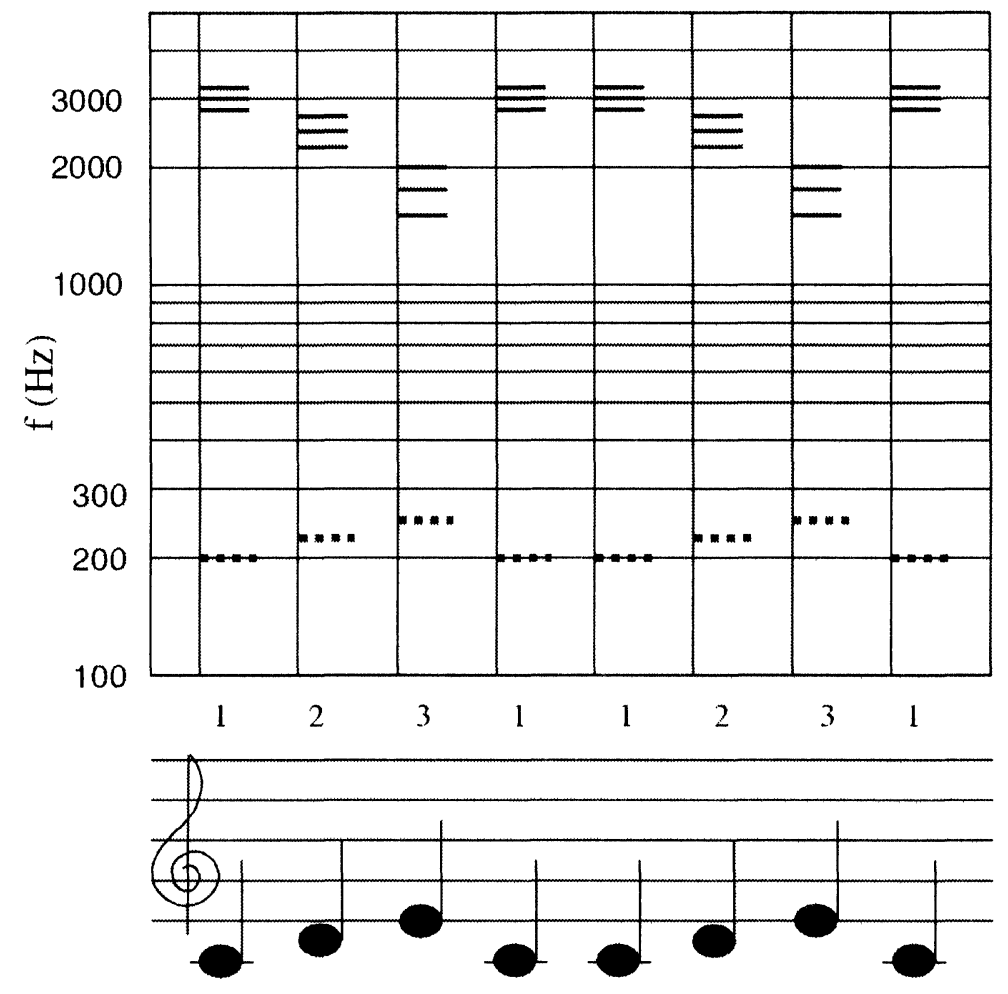

Fig. 1: Stimulus sequence of the virtual pitch melody. The sequence is built up of three different complex tones $\left(\mathrm{f}_{0}\right.$, tone 1: $200 \mathrm{~Hz}, \mathrm{f}_{0}$, tone2: $225 \mathrm{~Hz}$ and $\mathrm{f}_{0}$, tone3: $250 \mathrm{~Hz}$ ), played in the order 12311231 . The virtual melody follows the tune 'Frère Jacques' (lower inset: notation transposed to $C$ major, upper inset: dotted lines), whereas the spectral melody, given by three upper harmonics, has an inverse pitch contour (upper inset: solid lines).

The complex tones were composed by three harmonics of a missing fundamental frequency $\left(\mathrm{f}_{0}\right)$, which was set to 200,225 , and $250 \mathrm{~Hz}$, respectively. The melodic contour of the missing fundamentals followed the tune "Frère Jacques". The harmonics of the complex tones were selected in such a manner that their melodic contour was inverted with respect to the missing fundamental melody. Falling pitch steps in the virtual pitch sequence were associated with rising pitch steps in the spectral pitch sequence and vice versa (c.f. Fig. 1). When they perceive the spectral pitches, subjects hear a different melody compared with when they perceive the virtual pitches of the complex tones.
Therefore, the perceived pitch contour was used as an indicator of virtual pitch perception (i.e., the auditory Gestalt recognition). According to the selected tune, the three complex tones were presented in the order 12311231 (c.f. Fig. 1). Because of the constant duration of the included notes, no rhythmical cues were available that would facilitate the correct identification of the tune.

The predominance of one mode of pitch perception over the other one (spectral pitch vs. virtual pitch) has been found to depend on the spectral content of the complex tone and to differ inter-individually (Preisler, 1993). For this reason, two levels of difficulty were applied in this 
TABLE 1

Spectral composition of the three complex tones

\begin{tabular}{|c|c|c|c|}
\hline Tone & $\begin{array}{l}\text { Missing fundamental } \\
\text { frequency } \mathrm{f}_{0}[\mathrm{~Hz}]\end{array}$ & $\begin{array}{c}\text { Presented } \\
\text { harmonics [Hz] }\end{array}$ & $\begin{array}{c}\text { Ranks of } \\
\text { presented harmonics }\end{array}$ \\
\hline \multirow[t]{3}{*}{1} & \multirow[t]{3}{*}{200} & $3200(2800)$ & $16(14)$ \\
\hline & & $3000(2600)$ & $15(13)$ \\
\hline & & $2800(2400)$ & $14(12)$ \\
\hline \multirow[t]{3}{*}{2} & \multirow[t]{3}{*}{225} & $2700(2475)$ & $12(11)$ \\
\hline & & $2475(2250)$ & $11(10)$ \\
\hline & & $2250(2025)$ & $10(9)$ \\
\hline \multirow[t]{3}{*}{3} & \multirow[t]{3}{*}{250} & $2000(2000)$ & $8(8)$ \\
\hline & & $1750(1750)$ & $7(7)$ \\
\hline & & $1500(1500)$ & $6(6)$ \\
\hline
\end{tabular}

experiment. According to the predominance of low harmonics for virtual pitch perception (Plomp, 1967; Preisler, 1993; Ritsma, 1967), the easier level of difficulty contained harmonics that were, on average, one rank number lower than it was for the higher level of difficulty. The spectral regions of the constituent harmonics, making a switch in the type of perception likely, were proven in psychoacoustic pretests. The spectral composition of the three complex tones is displayed in Table 1.

During the MEG experiment, each tone was adjusted for loudness and was set to $60 \mathrm{~dB}$ above the individual hearing threshold $(60 \mathrm{~dB} \mathrm{SL}, \mathrm{SL}=$ sensation level). The duration of the complex tones was $150 \mathrm{~ms}$ with an inter-stimulus interval of $650 \mathrm{~ms}$. The time interval between sequences was $1500 \mathrm{~ms}$. The stimuli were masked by a continuous $500 \mathrm{~Hz}$ low-pass noise (filter slope: $40 \mathrm{~dB} /$ oct.) at an intensity of $30 \mathrm{~dB}$ SL to ensure that the perceived pitch was not due to combination tones resulting from nonlinear interactions in the auditory periphery.

Basically the harmonics of a complex tone can be of a resolved or an unresolved type. The term 
resolved means that the harmonics activate distinct regions (i.e. distinct auditory filter channels) along the cochlear partition. Unresolved harmonics elicit overlapping regions on the cochlea. To decide whether the stimuli used here are of the resolved or unresolved type, it is necessary to evaluate how many harmonics lie inside one single auditory filter channel. Based on the model of Moore \& Glasberg (1987), the harmonics of complex tones used here are of the unresolved type. Based on the model of Shackleton \& Carlyon (1994), however, the harmonics are partly resolved.

\section{PROCEDURE}

In pre-experimental sessions, the stimuli were presented to the subjects, who were asked to write down schematically the melody they perceived (the tune is going up or down). Subjects reporting to hear only the spectral pitch contour continued the experiment.

In the first MEG session, the stimuli were presented either to the left or to the right ear and auditory-evoked fields (AEFs) were recorded from the contralateral hemisphere. We presented 200 sequences per experimental condition ( 2 hemispheres $\times 2$ levels of difficulty). Thereafter, the subjects received a CD-ROM that contained repetitions of the melody. They were instructed to carefully listen to the CD for about 1 hour per day. Subjects were informed that there was a 'hidden', well-known tune behind the foreground tone sequence, which they should try to identify. Participants who did not succeed after 4 days of exposure at the more difficult level (c.f. Table 1) received a second CD-ROM with the less difficult version. The repeated exposure was terminated as soon as the test subject reported to hear the correct tune ("Frère Jacques"). After the switch in perception, a second MEG measurement was performed with stimuli of the same difficulty level, in which the perception of the virtual melody was successful. Thus, $2 \times 2$ (2 hemispheres $\mathrm{x}$ before and after virtual pitch perception) blocks with the same difficulty level for each subject were available for analysis. During MEG recordings, the subjects watched a self-selected silent video to keep them awake during the MEG measurement.

\section{ELECTROPHYSIOLOGICAL MEASUREMENTS AND DATA PROCESSING}

Magnetic field responses were recorded in a magnetically shielded room by means of a 37 . channel magnetometer (Magnes $^{\mathrm{TM}}$, Biomagnetic Technologies, San Diego, USA), positioned above the left or the right auditory cortex. The recording filter bandwidth was set to $0.1-100 \mathrm{~Hz}$. Data were sampled at a rate of $298 \mathrm{~Hz}$. For each hemisphere, AEFs to 200 stimulus sequences were recorded. After rejecting artifact-contaminated epochs, the stimulus-related epochs corresponding to tone 1 $(\mathrm{N} \cong 700)$ and tones 2 and $3(\mathrm{~N} \cong 175$, each) were averaged for each hemisphere.

\section{SOURCE ANALYSIS}

Source analysis, based on a single moving Equivalent Current Dipole (ECD) model, was applied to the N1m component of the slow AEF, peaking about $100 \mathrm{~ms}$ after stimulus onset, and to the evoked gamma-band response field. Source locations and strengths were estimated for every sample point of the averaged AEFs for the first $200 \mathrm{~ms}$ after stimulus onset. For the $\mathrm{N} 1 \mathrm{~m}$ component, data were low-pass filtered at $20 \mathrm{~Hz}$. For the gamma-band response field, data were band-pass filtered between 24 and $48 \mathrm{~Hz}$. Three sample points around the root mean square maxima (RMS) were chosen to obtain representative estimates of dipole parameters for $\mathrm{N} 1 \mathrm{~m}$ values. 
For gamma-band values, the parameters corresponding to the RMS maxima were used. The aim of the dipole analysis was to measure the center of activity corresponding to the main active areas of the auditory cortex and not to disclose all the different generator locations of the field. Therefore, a single ECD analysis was applied to the data. The estimated MEG sources were considered to be reliable when the following anatomical and physiological constraints were fulfilled: (a) the distance to the mid-sagittal plane was greater than $2 \mathrm{~cm}$, and (b) the minimal goodness of fit (gof) was $90 \%$ for the N1m and $85 \%$ for the gamma-band. Because of the low signal to noise $(\mathrm{S} / \mathrm{N})$ ratio of the GBRs, a sufficient gof was achieved only for tone 1 , in which case 4 times as many epochs had been collected (tone sequence: 12311231). Therefore, GBRs of tones 2 and 3 were not further analyzed. For the statistical evaluation of the experimental results, we performed an ANOVA on the data with hemisphere as an independent variable and virtual pitch perception as a repeated measurement factor. Thus, for each subject and each hemisphere, three datasets for $\mathrm{N} 1 \mathrm{~m}$ analysis and one dataset for gamma-band analysis were available.

\section{INDEPENDENT COMPONENT ANALYSIS (ICA)}

Multi-channel recorded auditory evoked GBRs were analyzed by means of ICA (Laubach et al., 1999, 2000). As a prerequisite for this type of analysis, a minimal $\mathrm{S} / \mathrm{N}$ ratio of 2 is required (Borschbach et al., 1999). The $\mathrm{S} / \mathrm{N}$ ratio was estimated by dividing the averaged absolute amplitudes of the GBR (20ms to $80 \mathrm{~ms}$ after the stimulus onset) by the averaged absolute amplitudes of the baseline activity $(-150 \mathrm{~ms}$ to $-50 \mathrm{~ms}$ before stimulus onset). Because the GBR is assumed to reflect the activity of a relatively wide cortical area (Pantev et al., 1991; Pantev, 1995),
ICA was used in a blind source separation framework to reveal the number of underlying independent components. The ICA does not solve the inverse problem. The goal of the ICA is to make the resulting components as independent as possible (Jung, et al., 2000, Vigario et al., 2000). This criterion of independence means that the spatial projections of components are different but fixed across time and that the source activations are temporally independent. Here the GBR is decomposed into independent components and the more spatio-temporal variability there is inherent in the data, the more independent components can be extracted by this method.

The ICA algorithm used here is based on the infomax principle, as formulated by Bell \& Sejnowski (1995). Thus, the data were separated into temporally independent and spatially stationary sources. Each independent source was projected back into the measurement sensor space to estimate its contribution to the measured data by calculating the norm error between the back-projected and the measured data. After ranking the components according to their contribution in a recursive way, the number of components required to explain the GBR within the time interval of 20 to $80 \mathrm{~ms}$ after stimulus onset was counted for a distinct error level. In the present study, the number of components necessary to explain the transient evoked GBR was compared by means of a paired sign-test for the spectral and virtual types of pitch perception.

\section{RESULTS}

Over both hemispheres, the SAEF and the transient GBR were recorded initially in subjects who were able to perceive only the spectral but not the virtual pitch melody. Two subjects recognized the virtual pitch melody "Frère Jacques" during the initial base-line MEG measurement and were 


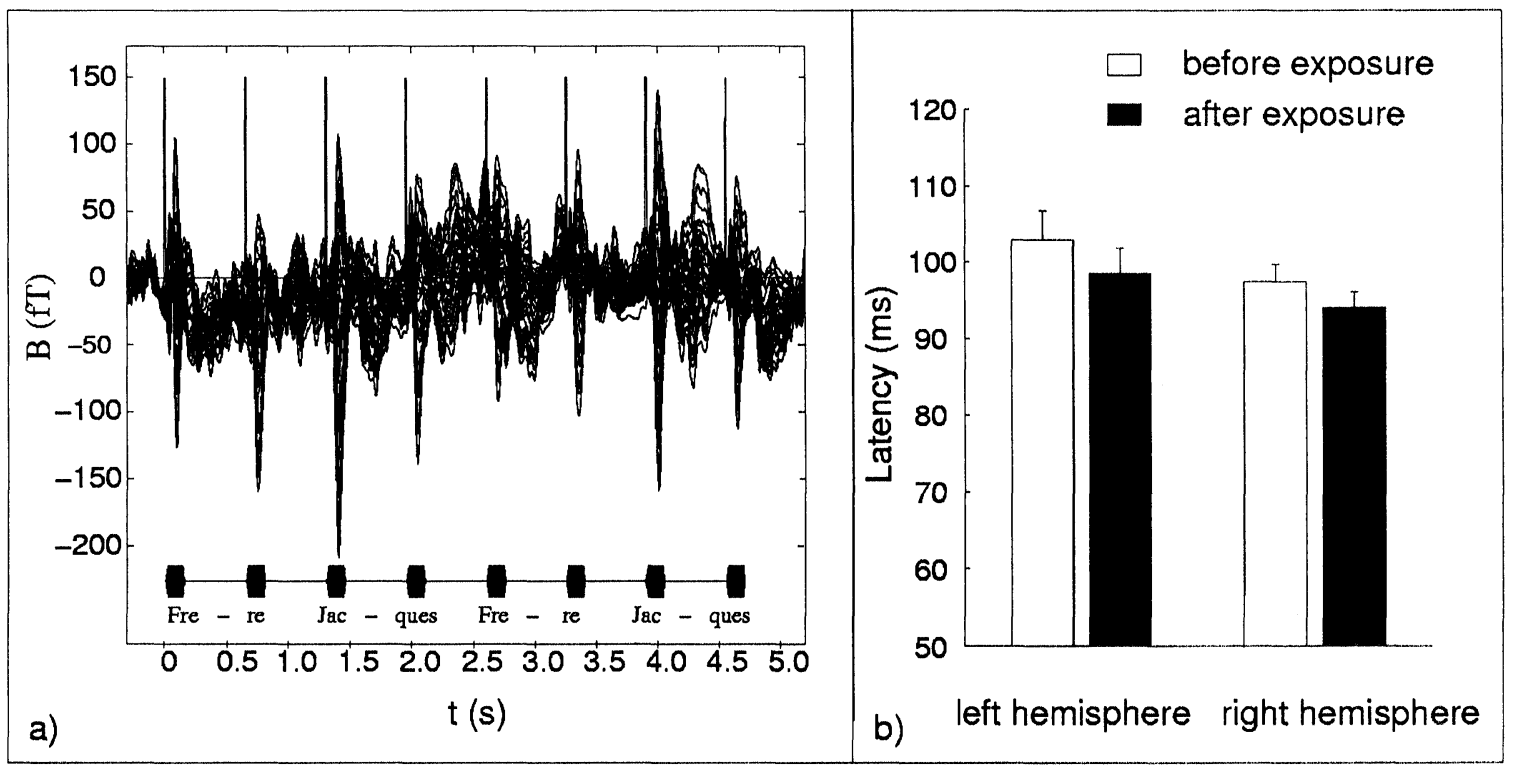

Fig. 2: (a) Averaged N1m-responses of one individual subject to the eight tone-sequences (magnetic fields in fT, low pass filtering: $0-20 \mathrm{~Hz}$ ). Waveforms, as derived from the 37 gradiometer channels, are shown together with the trigger signal (regular vertical lines). The inset denotes the onset and the duration of the stimuli. b) Mean values and standard errors of the mean (s.e.m.) of the latencies corresponding to the RMS maxima of wave $\mathrm{N} 1 \mathrm{~m}$. Black bars show the values after and white bars before exposure. Error bars illustrate the s.e.m.

excluded from further analysis. Seven test subjects recognized the virtual pitch melody "Frère Jacques" during 1 week of exposure, whereas one subject did not succeed after 1 week and was therefore excluded from further analysis.

Each test subject reported a sudden and highly impressive change in perception from the spectral to the virtual version of the melody. Four of the seven subjects perceived the virtual pitch at the more difficult level, (c.f. Table 1). Three subjects recognized the melody after four additional hours of exposure at the less difficult level. After successful virtual pitch perception, the sAEF and the GBR were measured again by applying exactly the same stimulus paradigm.

Fig. 2a illustrates the sAEF obtained from an individual subject. Each complex tone of the melody elicited a distinct $\mathrm{N} 1 \mathrm{~m}$. For source analysis of the SAEF, the data were considered for further evaluation only if the obtained gof values of the measured vs. the predicted field were at least $90 \%$ in the measurements before and after virtual pitch perception. For all subjects and both hemispheres, 16 of 21 possible data sets (three different complex tones and seven subjects) satisfying this condition were obtained for the left and 17 data sets for the right hemisphere. Statistical analysis (repeated measurements ANOVA) for the dipole moments and the source locations before and after virtual pitch perception did not indicate significant differences. In contrast, an effect on N1m latency was found. As shown in Fig. $2 b$ for all obtained data sets, N1m latency decreased for both hemispheres after virtual pitch 
perception. The N1m latency decreased from 102.9 $\pm 3.8 \mathrm{~ms}$ (mean to and standard error of the mean [s.e.m.]) to $98.5 \pm 3.3 \mathrm{~ms}$ for the left hemisphere and from $97.5 \pm 2.3 \mathrm{~ms}$ to $94.1 \pm 1.9 \mathrm{~ms}$ for the right hemisphere. Taking into account the pairs available from 'before' and 'after' measurements for the left and the right hemispheres, a significant mean latency difference of $3.87 \pm 1.29 \mathrm{~ms}$ was obtained $(\mathrm{DF}=1, \mathrm{p}=0.0057, \mathrm{~F}=8.8)$. The inter-hemispheric latency difference $(3.5 \pm 2.08 \mathrm{~ms})$ did not reach the level of significance $(\mathrm{DF}=1, \mathrm{p}=0.21, \mathrm{~F}=1.6)$.

For the evoked GBRs, waveforms of about 700 event-related epochs, corresponding to the complex tone 1 (c.f. Fig. 1), were averaged and filtered between 24 and $48 \mathrm{~Hz}$. GBRs obtained from four left and five right hemispheres in the corresponding sessions before and after the virtual pitch perception reached gof values of at least $85 \%$ (mean gof 'before': $94.1 \%$ and mean gof 'after': $93.8 \%$ ) and were considered for further source analysis. GBRs to tone 2 and tone $3 \mathrm{did}$ not reach the required $\mathrm{S} / \mathrm{N}$ ratio. Averaging GBRs to all three complex tones showed no sufficient improvement of the $\mathrm{S} / \mathrm{N}$ ratio for the datasets in which GBRs to tone 1 failed to fulfill the restraints in $\mathrm{S} / \mathrm{N}$ ratio. Therefore, GBRs to tone 2 and tone 3 were not considered for further source analysis. The respective averaged latencies of the RMS maximum of the GBR for the 'before' and 'after' virtual pitch perception conditions were $51.4 \pm 2.56 \mathrm{~ms}$ and $50.4 \pm 2.9 \mathrm{~ms}$. No significant difference was found in the gamma-band latency between the 'before' and 'after' measurements or between hemispheres.

Comparing the global field power (RMS value) between two measurements (i.e., before and after virtual pitch perception) is acceptable only if the position of the head with respect to the sensors is similar between the two measurements. Therefore, special efforts were made to achieve this relative constancy in sensor position. The distance of the central sensor coil to the midpoint of the head changed on average by $1.2 \mathrm{~mm}$ (s.e.m. $1.4 \mathrm{~mm}$ ).
Thus, the comparison of the global field power was appropriate. Individual data for GBRs for the conditions of 'before' and 'after' virtual pitch perception are displayed in Fig. 3a. It is evident that after switching to virtual pitch perception, GBRs appreciably increased. The averaged RMS values across subjects (Fig. 3b, upper panel) increased significantly from $6.2 \mathrm{fT}$ to $7.9 \mathrm{fT}$ for the left and from $4.96 \mathrm{fT}$ to $6.38 \mathrm{fT}$ for the right hemisphere (ANOVA: DF $=1, \mathrm{~F}=7.3, \mathrm{p}=0.031$ ). Similar behavior was also observed for the estimated cortical strength (dipole moment) of the GBR, which is independent of the sensor location. The cortical strength values were calculated for the RMS maximum of the GBR between 20 and $80 \mathrm{~ms}$ after stimulus onset (c.f. Fig. 3a). Figure $3 b$ (lower panel) illustrates that the estimated cortical strength of the gamma-band source significantly increased between the two corresponding sessions $(\mathrm{DF}=1, \mathrm{~F}=6.46, \mathrm{p}=0.039)$ from $1.03 \pm 0.16$ $\mathrm{nAm}$ to $1.92 \pm 0.71 \mathrm{nAm}$ in the left and from 1.59 $\pm 0.35 \mathrm{nAm}$ to $2.38 \pm 0.47 \mathrm{nAm}$ in the right hemisphere (c.f. Table 2). Between hemispheres, however, the differences did not reach statistical significance $(\mathrm{DF}=1, \mathrm{~F}=0.84, \mathrm{p}=0.39)$.

The locations of the gamma-band equivalent sources (means \pm standard error of the mean) obtained in the measurement sessions before and after virtual pitch perception are displayed in Table 2. Co-registering the GBF source locations with the subjects' MRIs indicated that the GBR areas are close to the anatomical structures of the primary auditory cortex (gyrus of Heschl). Group results averaged across subjects are illustrated in Fig. 4. Ellipses represent the standard errors of the means (s.e.m., inner ellipses) and the 95\% confidence intervals (outer ellipses) of the estimated source locations. Gamma-band sources are separated in the medial-lateral and inferiorsuperior direction, as the confidence intervals lie near or outside the corresponding mean values.

Accordingly, the ANOVA results show that 


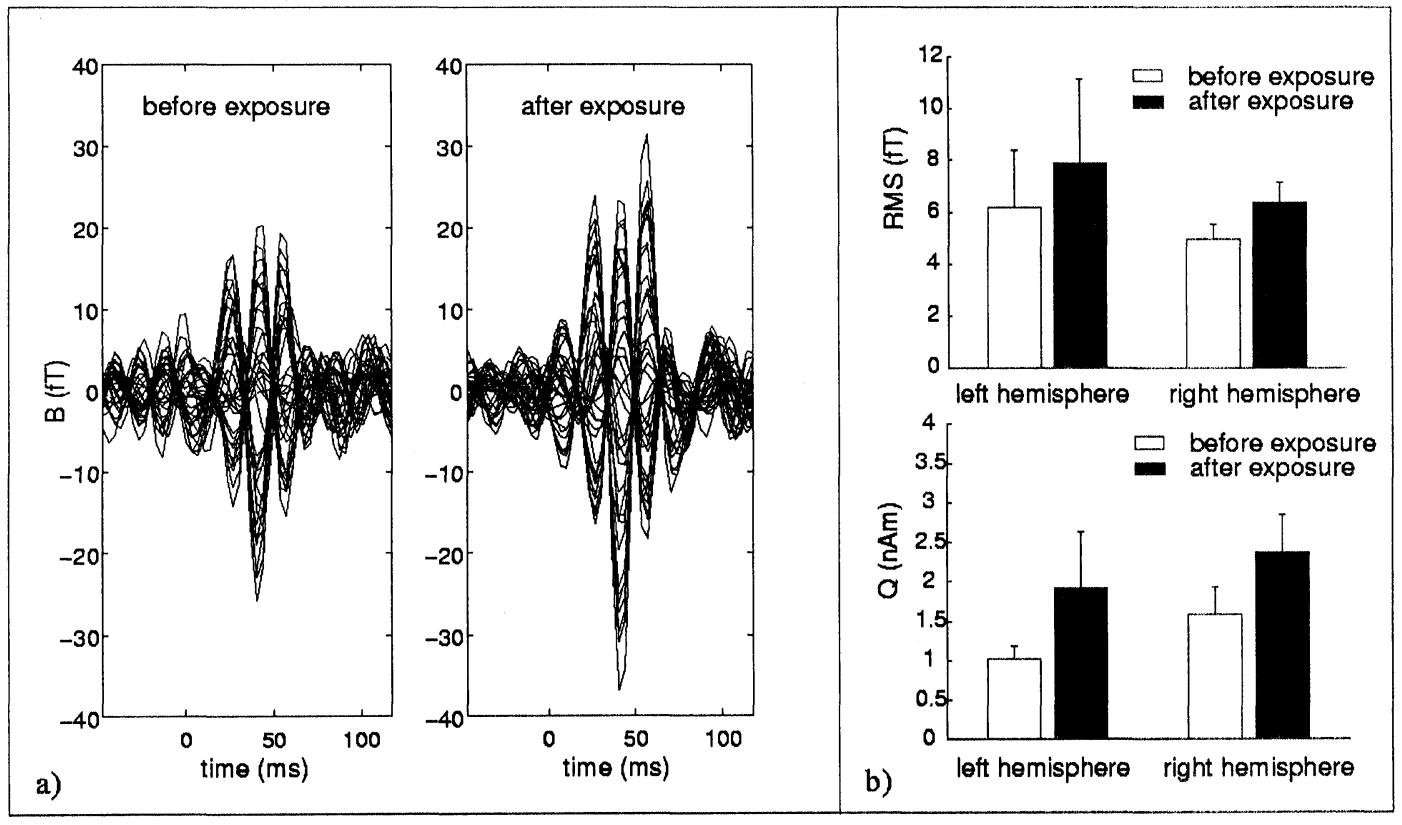

Fig. 3: (a) Gamma-band responses of one subject to the complex tone 1 of the sequence (band pass filtering: 24$48 \mathrm{~Hz}$ ). Data of the 37 measurement channels are plotted together for the session before (left) and the session after 1 week exposure (right). b) RMS values (top) and dipole moments of evoked gamma-band activity for the left and the right hemisphere, cross-averaged over all subjects. Black bars show the values after and white bars before exposure. Error bars indicate the s.e.m.

TABLE 2

Locations of the gamma-band equivalent sources (means \pm standard error of the mean) obtained in measurement sessions before and after virtual pitch perception

\begin{tabular}{|c|c|c|c|c|}
\hline \multirow{2}{*}{} & \multicolumn{2}{|c|}{ Left hemisphere } & \multicolumn{2}{c|}{ Right hemisphere } \\
\cline { 2 - 5 } & Before & After & Before & After \\
\hline$X[\mathrm{~cm}]$ & $2.26 \pm 0.83$ & $1.38 \pm 0.41$ & $1.16 \pm 0.24$ & $1.50 \pm 0.48$ \\
\hline $\mathrm{Y}[\mathrm{cm}]$ & $5.27 \pm 0.68$ & $4.61 \pm 0.30$ & $-4.56 \pm 0.50$ & $-4.05 \pm 0.61$ \\
\hline$Z[\mathrm{~cm}]$ & $6.73 \pm 0.58$ & $6.01 \pm 0.69$ & $5.39 \pm 0.27$ & $4.69 \pm 0.56$ \\
\hline $\mathrm{Q}[\mathrm{nAm}]$ & $1.03 \pm 0.16$ & $1.92 \pm 0.71$ & $1.59 \pm 0.35$ & $2.38 \pm 0.47$ \\
\hline RMS [fT] & $6.20 \pm 2.2$ & $7.91 \pm 3.20$ & $4.95 \pm 0.59$ & $6.38 \pm 0.79$ \\
\hline
\end{tabular}



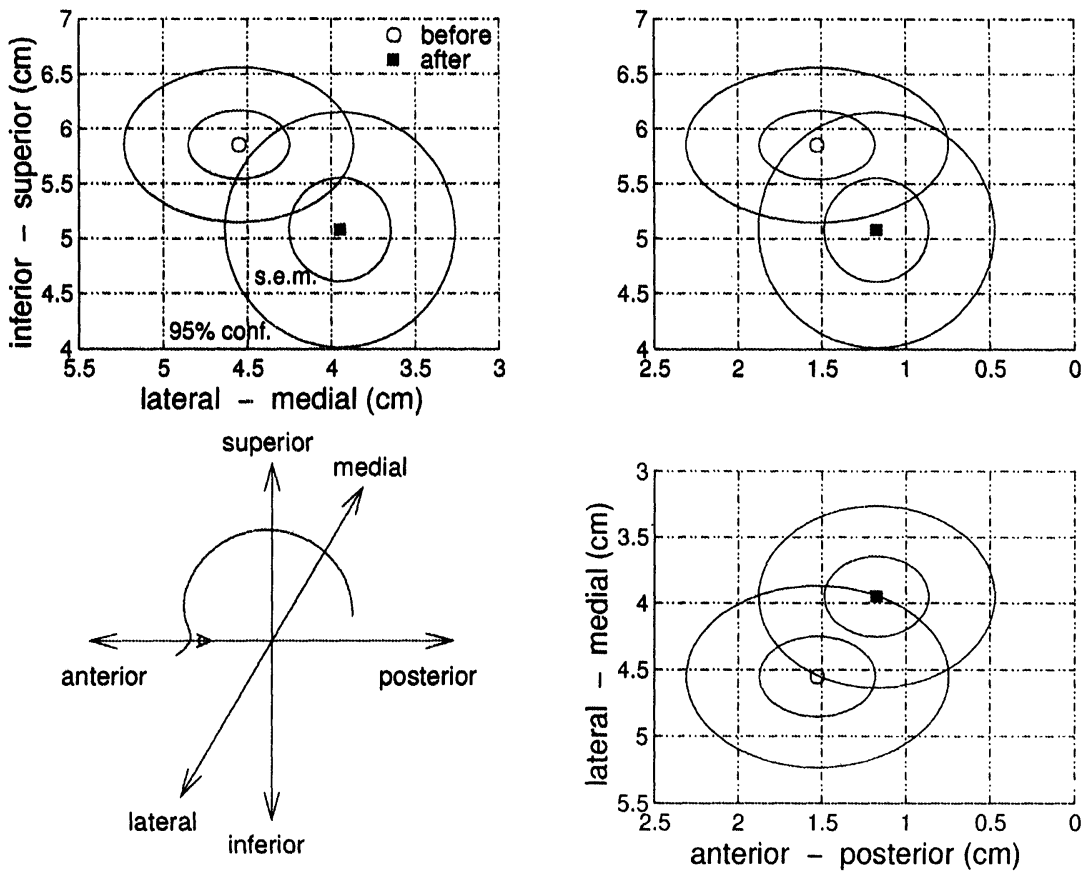

Fig. 4: Mean GBR source locations before and after exposure collapsed over all subjects and both hemispheres. The inner circles illustrate s.e.m. and the outer circles denote the corresponding $95 \%$ confidence intervals.

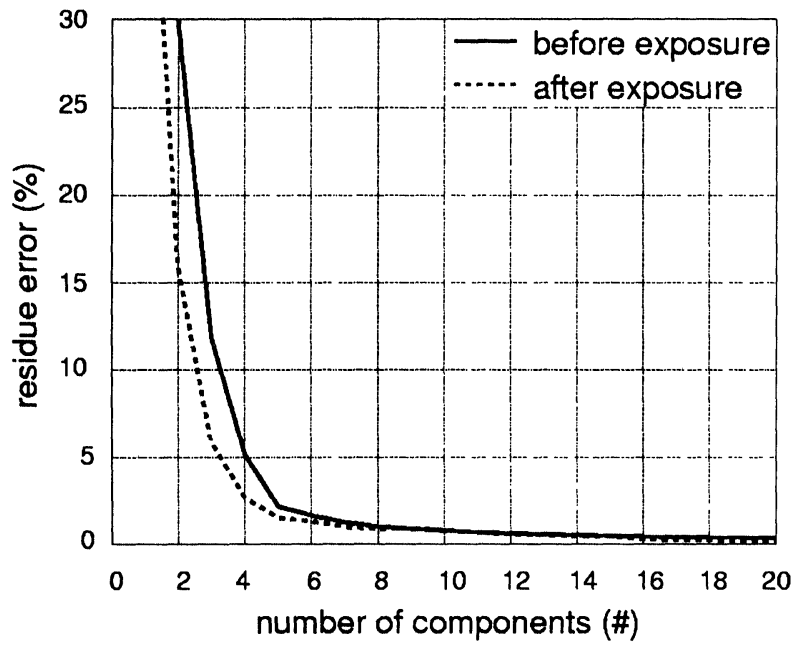

Fig. 5: Results of ICA analysis from a dataset of one individual subject. The curves illustrate the number of components necessary to explain the evoked gamma-band activity for different levels of error percentages. Over an entire range of error levels, more components are needed to explain the data before (solid line) than after exposure (dashed line). 
after virtual pitch perception GBR sources were located significantly more medially $(\mathrm{DF}=1$, $\mathrm{p}=0.033, \mathrm{~F}=6.99$ ) by about $0.5 \mathrm{~cm}$ as compared with GBR source activity before virtual pitch perception. The mean difference of $0.6 \mathrm{~cm}$ in the inferior-superior direction was close to significance $(D F=1, p=0.061, F=4.96)$, whereas no significant difference was found in the anterior-po te: ior direction ( $\mathrm{DF}=1, \mathrm{p}=0.43, \mathrm{~F}=$ 0.7).

The recorded GBRs in this study underwent an additional evaluation by means of independent component analysis (ICA). Five pairs of left and five pairs of right hemispheric data sets reached the required $\mathrm{S} / \mathrm{N}$-ratio of at least 2 . Gamma-band data were separated into independent components. These components were then projected back into the sensor space to evaluate the influence of each independent component on the measured gammaband data in the time range of 20 to $80 \mathrm{~ms}$ after stimulus onset. Fig. 5 shows the results of the ICA for one subject. The curves illustrate up to which error level the measured data are explained by a distinct number of independent components. Before virtual pitch perception (solid line), more independent components were needed to explain the GBR than after (dashed line). The S/N-ratio also increased, due to the increase of RMS values after virtual pitch perception. To avoid an overinterpretation in terms of an increase of S/N-ratio, we randomly excluded epochs from the averaging process to reduce the $\mathrm{S} / \mathrm{N}$-ratio in the "after virtual pitch perception datasets", in which the ratio was normally increased (this was the case in 7 of 10 datasets). After this procedure the $\mathrm{S} / \mathrm{N}$-ratio, averaged across subjects, decreased from $4.22 \pm$ 1.91 to $4.18 \pm 1.9$ for the 'before' to 'after' virtual pitch-perception measurements, respectively. Even then the ICA results, averaged across subjects and hemispheres, clearly showed that significantly fewer components are needed to explain the gamma-band fields for a wide range of error levels after virtual pitch perception. This difference was statistically significant at $p<0.025$ for the levels of $4 \%$ and $7 \%$ and at $p<0.05$ for levels $3 \%, 6 \%$, and $8 \%$.

\section{DISCUSSION}

In this study the influence of different pitch perceptions (spectral versus virtual) of the same stimulus on the auditory cortex, contralateral to the ear of presentation, was investigated for both hemispheres. The type of perception of the subjects switched from a spectral to virtual mode, due to the exposure of about 1 week. A strong increase was found for the global field power of the gammaband response (GBR) and its corresponding cortical strength. The sources of the equivalent current dipoles after switch of perception were located about $6.6 \mathrm{~mm}$ more medially for the left and $5.1 \mathrm{~mm}$ for the right hemisphere as compared with the corresponding sources before the switch. The ICA analysis showed that a smaller number of independent components were necessary to explain the GBRs after switch in perception.

To our knowledge, the present study is the first systematic investigation directly addressing the question of learning-induced plasticity during virtual pitch perception of complex tones. Test subjects were able to 'learn' to perceive the virtual pitch of the complex tones. All subjects reported that recognition of the virtual melody occurred abruptly - analogous to visual Gestalt recognition (Tallon-Baudry \& Bertrand, 1999)-and that it was irreversible. This result corresponds to casual observations by Deutsch \& Steger (1978), who reported that some of the spectral listeners could be trained to perceive virtual pitch, whereas reversed training in virtual listeners had no effect. Such one-directional learning indicates that once it has been recognized, a Gestalt-like percept cannot be ignored. There are indications from infant studies that virtual pitch abstraction abilities may 
be gained in early childhood. Specifically, it has been found that 7-month-old but not 4-month-old babies could categorize tonal stimuli according to their virtual pitches (Clarkson \& Clifton, 1984; Bundy \& Colombo, 1982). Therefore, based on the more elementary mode of spectral pitch perception, virtual pitch sensations are likely to reflect higher-order abstraction processes.

Few findings have been reported, suggesting indirectly that plastic reorganization processes are involved in pitch perception. Using ambiguous two-tone sequences, Smoorenburg (1970) and Deutsch \& Steger (1978) observed a high interindividual variability of the predominant mode of pitch perception. Also, Preisler (1993) found that the ability to extract virtual pitch in skilled musicians is more refined than in non-musicians. These observations demonstrate that the perceived pitch depends not only on the physical properties of the stimulus but also on the individual's abilities. Nevertheless, determining to what extent these inter-individual differences are due to experience and to inherited factors remains difficult. Our present results, however, directly indicate that learning induced plasticity may be involved in the perception of the pitch of complex tones.

After repeated exposure to the virtual pitch melody, the GBF sources were located more inferior and significantly more medial as compared with the sources measured before repeated exposure. That the shift in location is the result of a tonotopic effect is unlikely. Earlier experiments with a stimulus paradigm designed to study the tonotopic representation of gamma-band sources have shown no tonotopic gradient for the sources of auditory GBRs (Pantev \& Elbert, 1994, Bertrandt \& Pantev, 1994). Also, a change of the quality of the dipole fit for the measurements before and after exposure is unlikely because we did not observe any relevant change in the gof $(94.1 \%$ before and $93.8 \%$ after exposure) between the before and after conditions.

The observed shift in GBR source location might indicate that, despite the presentation of the same stimulus, different areas of the cortical network were involved in its processing after the switch in perception. Alternatively, this shift might indicate that distinct areas are at least more intensively involved in the generation of the GBR after the switch in perception than before.

A significant increase of gamma-band cortical strength of more than $50 \%$, which was associated with a corresponding change of global field power, was observed after the switch in perception. Additionally, the observation that significantly fewer ICA components were needed to explain the gamma-band activity after the switch in perception demonstrates that the stimuli were processed in a different way. In visual experiments, comparable effects have been repeatedly observed for the time variant-induced gamma-band activity (Singer \& Gray, 1995; Tallon-Baudry \& Bertrand 1999). For instance, in a visual pattern recognition experiment (Tallon-Baudry et al., 1997b), gamma-band oscillations were found to be significantly increased after the foreground physical features had been integrated into a known visual object. A basic rule of the binding theory is that during Gestalt recognition, the different features of an object are combined into a coherent percept. In the present experiment, the switch from the analytic to the synthetic mode of pitch perception was invoked by a convergence of harmonically related information. The ICA results obtained here can be interpreted to reflect this convergence, which was achieved by a higher synchronization of the involved cortical areas. This higher degree in synchronization could indicate a change in information flow between different cortical areas and might explain the shift in location found for the gamma-band sources. This type of auditory Gestalt recognition was associated with plastic 
reorganization processes, expressing themselves as an increase of the global field power and cortical strength. The increase may be interpreted either by a higher degree of synchronization, as already suggested by the ICA, by enlargement of the involved cortical area, or most probably by both.

In contrast to the GBRs, no differences in location and strength were observed for the estimated Nim sources, which are expected to originate from the secondary auditory cortex (Pantev et al. 1995, Lütkenhöner \& Steinsträter, 1998, Engelien et al, 2000). This result might indicate that the plastic reorganization due to the switch in perception affects the primary auditory area only, or at least more than the secondary ones.

Knief et al. (2000) observed functional differences in the GBR, due to the perception of coherent versus non-coherent auditory stimuli. In that study, the harmonics were spectrally resolved in the cochlea, which means that the harmonics of the complex tone activate distinct regions along the cochlear partition. In the present experiment, higher harmonics were chosen, thus the harmonics were not spectrally resolved and the auditory system had to extract the fundamental frequency from the temporal information. Thus, the results of the former study have been corroborated in the present study for unresolved or for partly resolved spectra (c.f. Experimental sec.). Whether temporal information, spectral information, or both are used to extract the virtual pitch in the former study is not clear. If resolved and unresolved harmonics are processed by different mechanisms is still a matter of discussion in the literature (Meddis \& O'Mard, 1997; Shackleton \& Carlyon, 1994; Carlyon \& Shackleton, 1994). The results of our previous and the present study indicate, however, that in both cases the evoked GBRs represent functional correlates of subjective pitch sensations. For both types of stimuli, the ICA results indicate that the perception of virtual pitch leads to a higher degree of synchronization of the involved cortical regions.

Surprisingly, in the present experiment, no differences in the GBR were found between the hemispheres. This was also the case for both the equivalent cortical sources and the number of independent components. A few studies have explicitly addressed the question of a possible lateralization of pitch perception of the missing fundamental. Zatorre (1988) reports that temporal lobectomy of the right but not the left hemisphere affects this perception. Our findings indicate that, at the level of the primary auditory cortex, no clear hemispheric lateralization exists for the processing of virtual pitch. Because it is likely that temporal information was relevant for the virtual pitch extraction, our observations are in line with the results of a PET-study by Griffiths et al. (1998). In that study, no hemispheric differences were observed at the level of the A1, similar to the findings of the present study. In contrast, the latency of the N1m-component showed a slight hemispheric effect that failed to reach significance. The direction of this difference is comparable to the results of Paquette et al. (1996), who reported significantly shorter response times for missing fundamental stimuli presented to the left as compared with the right ear, thus suggesting right hemispheric advantage.

In conclusion, the results of the present study indicate that transient evoked gamma-band activity can be regarded as a correlate of a virtual pitch formation process for both resolved and unresolved harmonics. Thus, non-invasive recording of gamma-band activity provides us with the opportunity to explain the mechanisms underlying the auditory binding processes and the auditory Gestalt recognition in humans. In the present study, the change of gamma-band fields indicates plastic functional reorganization of the human auditory cortex corresponding to the switch from spectral to virtual pitch perception. 


\section{ACKNOWLEDGEMENTS}

This work was supported by the Deutsche Forschungsgemeinschaft, $\mathrm{Pa}$ 392/4-2. We thank Karin Berning for technical assistance and Laurel J. Trainor and David I. Shore for helpful comments on the manuscript.

\section{REFERENCES}

Beerends J. 1989. The influence of duration on the perception of pitch in single and simultaneous complex tones. J Acoust Soc Am 86: 1835-1844.

Bell A, Sejnowski TW. 1995. An information maximization approach to blind separation and blind deconvo-lution. Neural Comput 7: 1129-159.

Bertrand O. 1998. Auditory induced $40-\mathrm{Hz}$ activity during a frequency discrimination task. NeuroImage $7: 370$.

Bertrandt O, Pantev C. 1994. Stimulus frequency dependence of the transient oscillatory auditory evoked response $(40 \mathrm{~Hz})$ studied by electric and magnetic recordings in humans. In: Pantev $C$, Elbert T, Lütkenhöner B, eds, Oscillatory EventRelated Brain Dynamics. New York, NY, USA; London, UK: NATO ASI Series; 231-242.

Bharucha J, Tramo M, Zatorre R. 1993. Abstraction of the missing fundamental following bilateral lesions of the auditory cortex. Soc Neurosci Abstr 1687.

Borschbach M, Schulte M, Knief A, Lippe WN, Pantev C. 1999. Aspects of neuronal learning rules for the blind separation of independent brain activities. ICS'99, Santa Barbara, California, USA; 143-144.

Bundy RS, Colombo J. 1982. Pitch perception in young infants. Dev Psychol 18: 10-14.

Carlyon RP, Shackleton TM. 1994. Comparing the fundamental frequencies of resolved and unresolved harmonics: Evidence for two pitch mechanisms? J Acoust Soc Am 95: 3541-3554.

Clarkson MG, Clifton RK. 1984. Infant pitch perception: Evidence for responding to pitch categories and the missing fundamental. J Acoust Soc Am 77: 1521-1528.

Deutsch W, Steger R. 1978. Binaurales Residuum versus Lokalisation. Mitteilungen der Kommission für Schallforschung 5: 41-53.
Engelien A, Schulz M, Ross B, Arolt V, Pantev C. 2000. A combined functional in vivo measure for primary and secondary auditory cortices. Hearing Res 148: 153-160.

Griffiths TD, Buchel C, Frackowiak RS, Patterson RD. 1998. Analysis of temporal structure in sound by the human brain. Nat. Neurosci 1: 422-427

Hartmann WM. 1988. Pitch perception and the segregation and integration of auditory entities. In Edelman GM, Gall WE, Cowan WM, ed, Auditory Function. New York, NY, USA: Wiley; 623-645.

Jung TP, Makeig S, Lee TW, McKeown MJ, Brown G, Bell AJ, et al. 2000. Independent Component Analysis of Biomedical Signals. In: Proc. $2^{\text {nd }}$ Int Workshop on Independent Component Analysis and Signal Separation; 633-644.

Knief A, Schulte M, Bertrand O, Pantev C. 2000. The perception of coherent and non-coherent auditory objects: a signature in gamma frequency band. Hearing Res 145: 161-168.

Laubach M, Shuler M, Nicolelis MAI. 1999. Independent component analyzes for quantifying neuronal ensemble interactions. J Neurosci Meth 94: 141-154.

Laubach M, Wessberg J, Nicolelis MAI. 2000. Cortical ensemble activity increasingly predicts behavioral outcomes during learning of a motor task. Nature 405: 567-571.

Lütkenhöner B, Steinsträter O. 1998. High-precision neuromagnetic study of the functional organization of the human auditory cortex. Audiol Neurootol 3: 191-213.

Meddis R, O'Mard L. 1997. A unitary model of pitch perception. J Acoust Soc Amer 102: 1811-1820.

Moore BCJ, Glasberg BR. 1987. Formulae describing frequency selectivity as a function of frequency and level, and their use in calculating excitation patterns. Hearing Res 28: 209-225.

Oldfield RC. 1971. The assessment and analysis of handedness: The Edinburgh inventory. Neurophysiologia 9: 97-113.

Pantev C, Makeig S, Hoke M, Galambos R, Hampson S, Gallen C. 1991. Human auditory evoked gammaband magnetic fields. Proc Natl Acad Sci USA 88: 8996-9000.

Pantev C. 1995. Evoked and induced gamma-band activity of the human cortex. Brain Topography 7 : 321-330.

Pantev C, Elbert T. The transient auditory evoked gamma-band field. 1994. In: Pantev C, Elbert T, Lütkenhöner B, eds, Oscillatory Event-Related 
Brain Dynamics. New York, NY, USA; London, UK: NATO ASI Series; 219-230.

Pantev C, Bertrand O, Eulitz C, Verkindt C, Hampson S, Schuierer G, et al. 1995. Specific tonotopic organization of different areas of the human auditory cortex revealed by simultaneous magnetic and electric recordings. Electroencephalogr Clin Neurophysiol 94: 26-40.

Paquette C, Bourassa M, Peretz I. 1996. Left ear advantage in pitch perception of complex tones without energy at the fundamental. Neuropsychologia 34: 153-157.

Plomp R. Pitch of complex tones. 1967. J Acoust Soc Am 41: 1526-1533.

Preisler A. 1993. The influence of spectral composition of complex tones and of musical experience on the perceptibility of virtual pitch. Percept Psychophys 54: 589-603.

Pulvermüller F, Eulitz C, Pantev C, Mohr B, Feige B, Elbert T, et al. 1996. High-frequency cortical responses reflect lexical processing: An MEG study. Electro-encephalogr Clin Neurophysiol 98: 76-85.

Ritsma RJ. 1967. Frequencies dominant in the perception of the pitch of complex sounds. J Acoust Soc Am 42: 191-198.

Seebeck A. 1843. Über die Sirene. Ann Phys Chem 60: 449-481.

Shackleton TM, Carlyon RP. 1994. The role of resolved and unresolved harmonics in pitch perception and frequency modulation discrimination. J Acoust Soc Am 95: 3529-3540.

Singer W, Gray CM. 1995. Visual feature integration and the temporal correlation hypothesis. Ann Rev
Neurosci 18: 555-586.

Smoorenburg GF. 1970. Pitch perception of twofrequency stimuli. J Acoust Soc Am 4: 924-942.

Tallon-Baudry C, Bertrand O. 1999. Oscillatory gamma activity in humans and its role in object representation. Trends Cogn Sci 3: 151-162.

Tallon-Baudry C, Bertrand O, Delpuech C, Pernier J. 1996. Stimulus specificity of phase-locked and non-phase-locked $40 \mathrm{~Hz}$ visual responses in human. J Neurosci 16: 4240-4249.

Tallon-Baudry C, Bertrand O, Wienbruch C, Ross B, Pantev C. 1997a. Combined EEG and MEG recordings of visual $40 \mathrm{~Hz}$ responses to illusory triangles in human. NeuroReport 8: 1103-1107.

Tallon-Baudry C, Bertrand O, Delpuech C, Pernier J. 1997b. Oscillatory gamma-band $(30-70 \mathrm{~Hz})$ activity induced by a visual search task in human. J Neurosci 17: 722-734.

Tallon-Baudry C, Bertrand O, Peronnet F, Pernier J. 1998. Induced gamma-band activity during the delay of a visual short-term memory task in humans. J Neurosci 18: 4244-4254.

Terhardt E. 1974. Pitch, consonance, and harmony. J Acoust Soc Am 55: 1061-1069.

Vigario R. 2000. Independent Component Approach to the Analysis of EEG and MEG Recordings. IEEE Trans Biomed Eng 47: 589-593.

Winkler I, Tervaniemi M, Näätänen R. 1997. Two separate codes for missing-fundamental pitch in the human auditory cortex. J Acoust Soc Am 102: 1072-1082.

Zatorre RJ. 1988. Pitch perception of complex tones and human temporal-lobe function. $\mathbf{J}$ Acoust Soc Am 84: 566-572. 

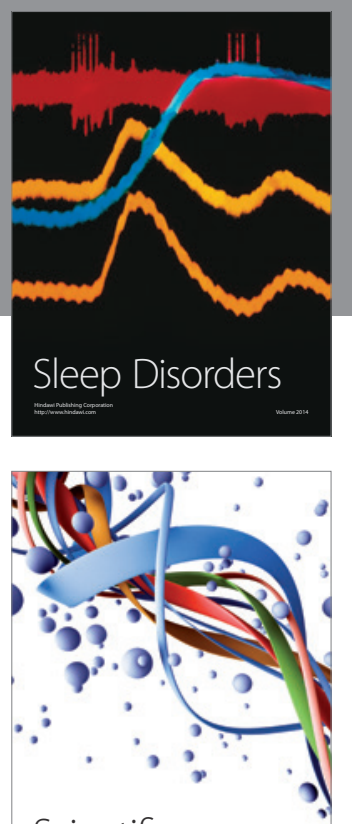

Scientifica
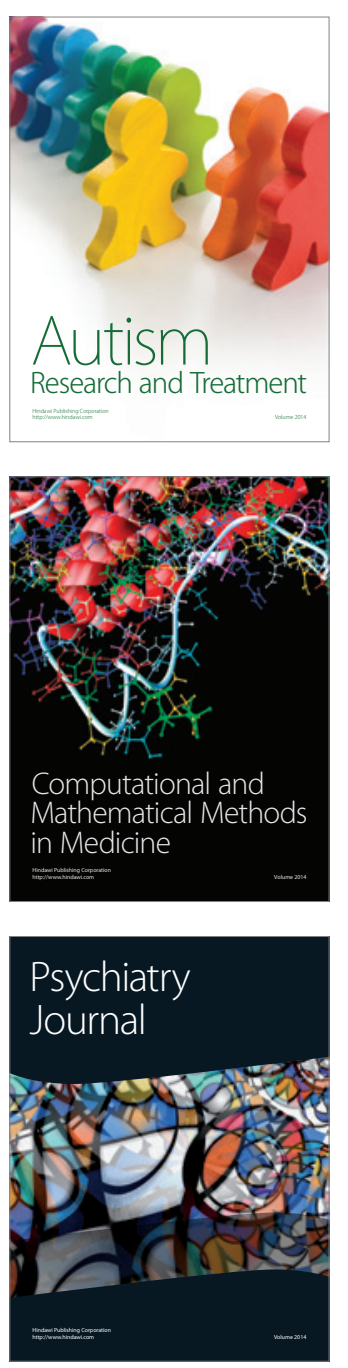
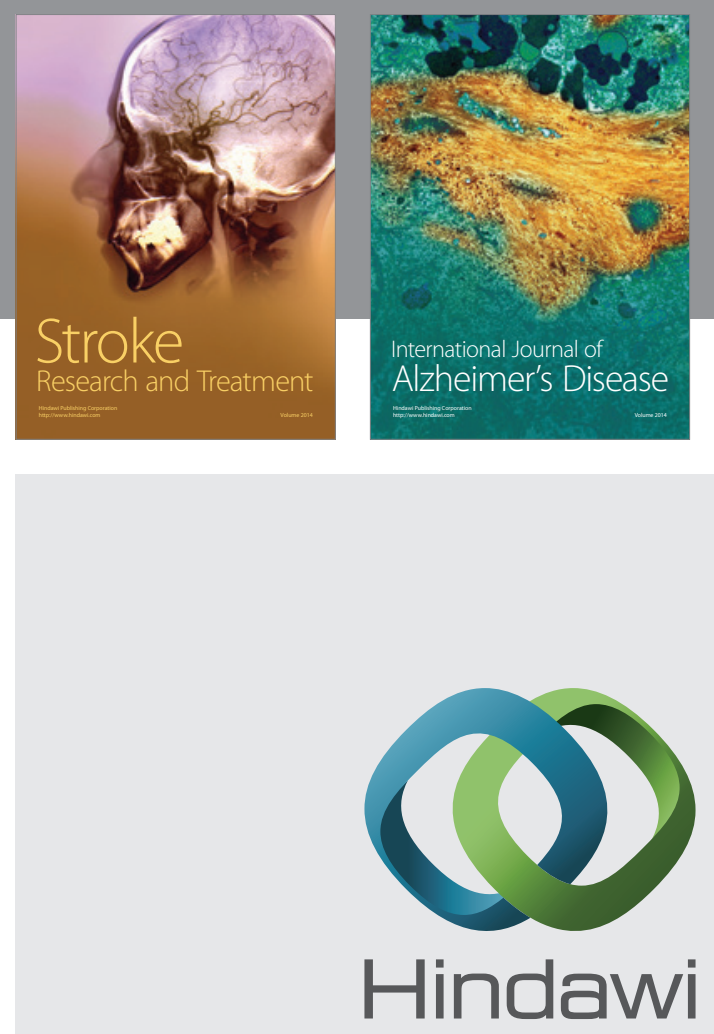

Submit your manuscripts at

http://www.hindawi.com
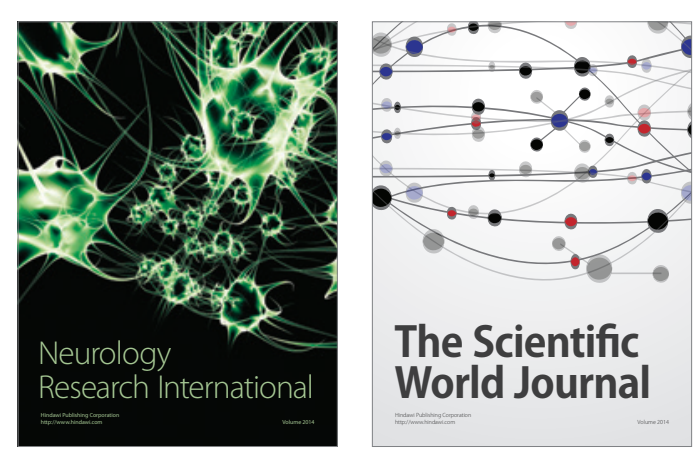

The Scientific World Journal

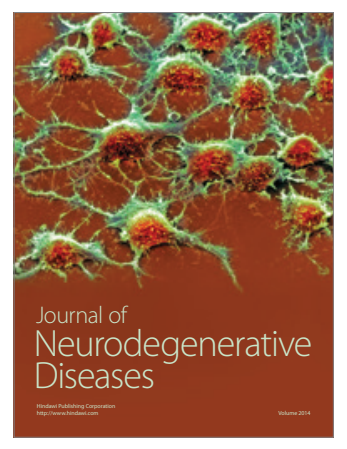

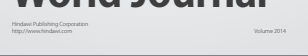

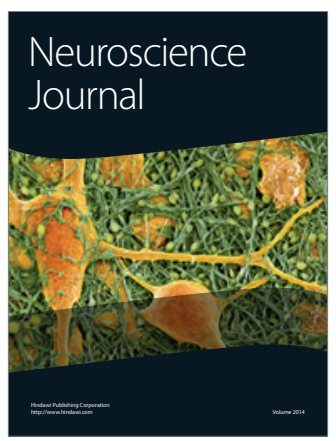

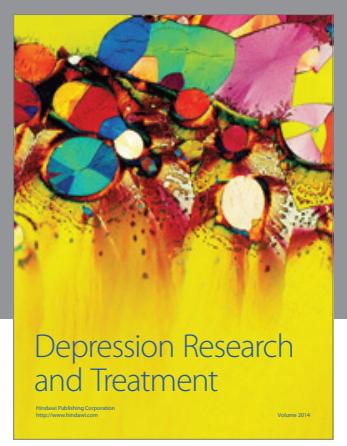
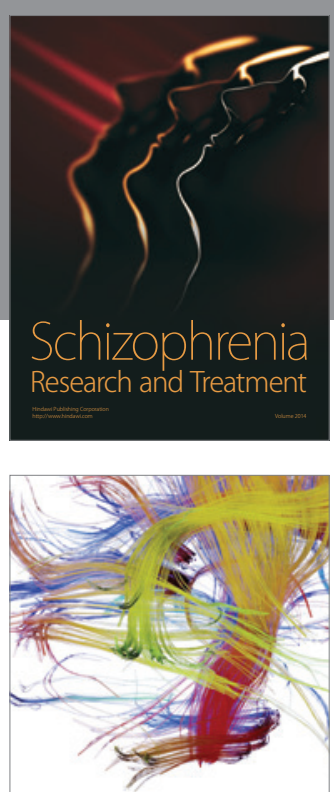

Brain Science

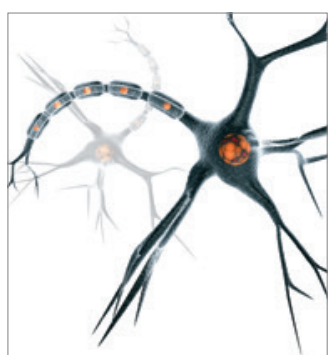

Neural Plasticity
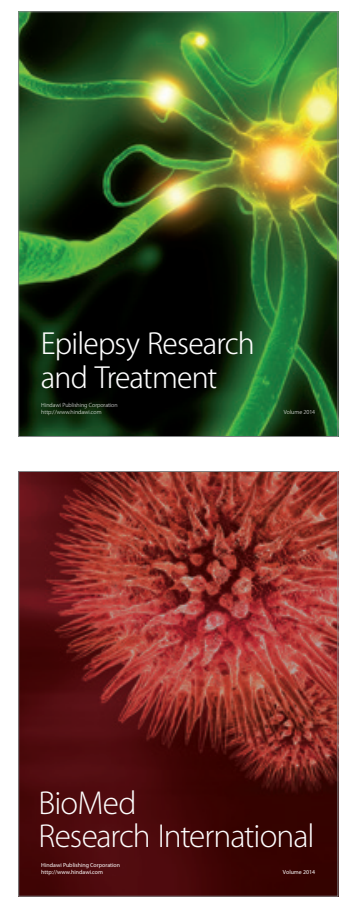

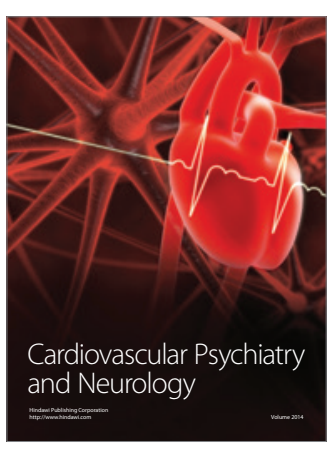

Parkinson's

Disease
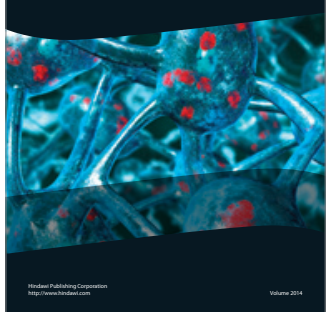